\title{
Differential Effect of Antibody Removal on ABO Blood Group Type Chain Specific Antibodies Over Time
}

Andrew Bentall ${ }^{1}$, Jeya Mylvaganam², Jean Pearcey², Bruce Motyka², Chris Cairo3 ${ }^{3}$, Todd Lowary ${ }^{3}$, Jillian Buriak $^{4}$, Lori West ${ }^{2}$ and Simon Ball ${ }^{1}$

1Department of Nephrology, University Hospital Birmingham, Birmingham, United Kingdom; 'Departments of Pediatrics, Surgery, and Immunology, University of Alberta, Alberta Transplant Institute, Edmonton, Canada; ${ }^{3}$ Alberta Glycomics Centre,, Univ of Alberta Dept of Chemistry, Edmonton, Canada and ${ }^{4}$ Dept of Chemistry, National Institute of Nanotechnology., Edmonton, Canada.

\section{BACKGROUND AND AIMS}

Incompatible registry data find poorer outcomes in ABO incompatible kidney recipients((ABOi), but $A B O i$ remains a potentially important treatment particularly for blood group 0 recipients.

Outcomes are reported independently of the antibody titre, however there is significant varation in titre measurement.

The trisaccharide epitope defines A and B blood group antigens where antibody can bind to subtype chains (I-VI).

Only subtype II is expressed on cardiac vascular endothelium, which is also expressed on glomerular endothelium.

Following pediatric ABO-incompatible heart transplantation (ABOiHTX) there is evidence of humoral tolerance to subtype II but not other allograft blood group antigen subtypes.

This study reports subtype-specific antibody quantification in adult $\mathrm{ABO}$ idney transplant (ABOiKTx) recipients.

\section{METHODS}

From a previous published ABOi kidney transplant cohort, samples from pre-antibody removal, day of transplantation and at a late time point (6 or 12 months) were measured by a central haemagglutination (HA) assay and by a published microarray platform.

Antibody binding to ABH subtype antigens I-VI was quantified using a microarray platform in which the mean fluorescence intensity (MFI) of binding is proportionate to the concentration of antibody. The assay assessed IgM and IgG binding.

Plasma samples were obtained from 68 ABOiKTx recipients with blood group $A$ donors - both $O$ and $B$ recipients

Patients received either plasma exchange or A-trisaccharide specific antibody removal (Glycosorb) columns for removal of anti-A antibodies.

Data were analysed by change in antibody measurement with treatment and over time with respect to specific blood group antigen subtype binding.

\section{RESULTS}

IgG anti-A subtype specific binding in O recipients of A-mismatched kidneys before and after plasma exchange or immunoadsorption

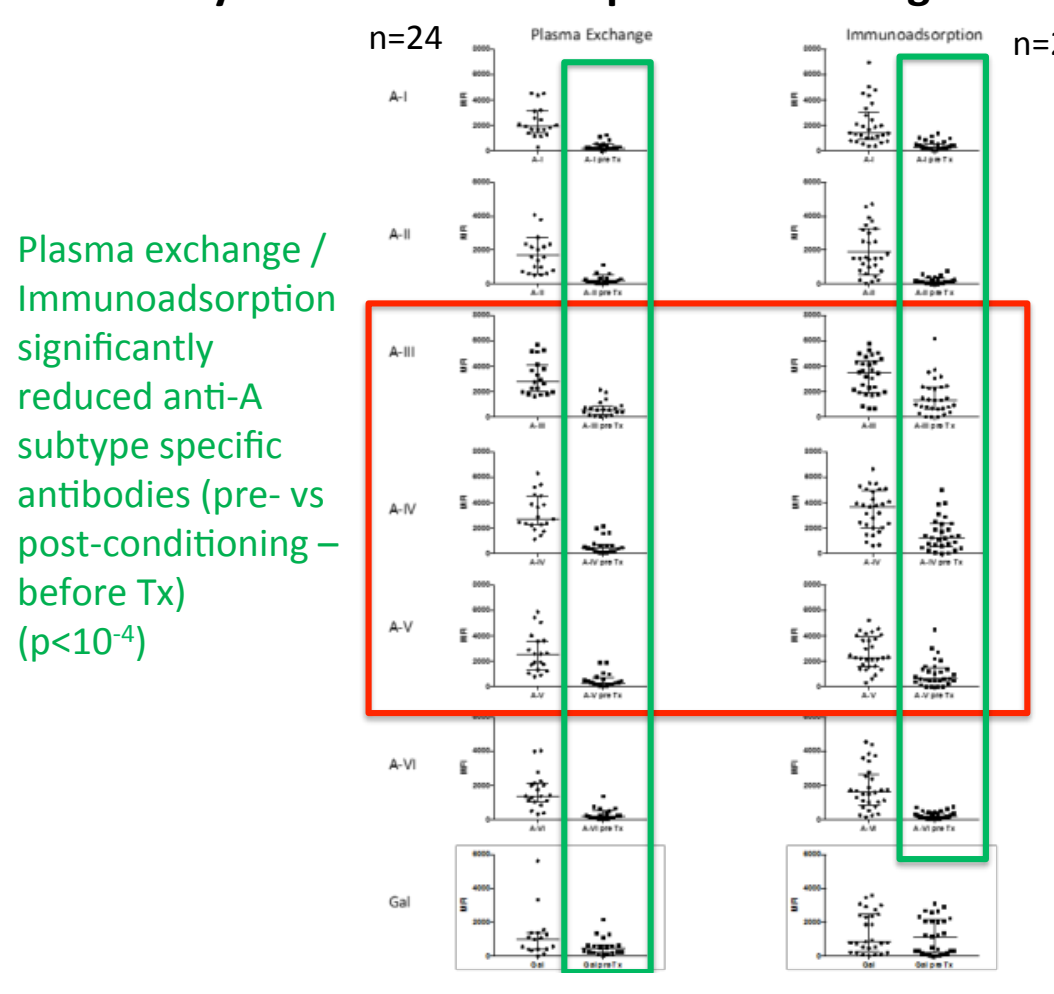

Pre-treatment to post EART less subtype III and IV reduction in IA than PEX. There was pan IgG removal in PEX, no Gal removal in IA

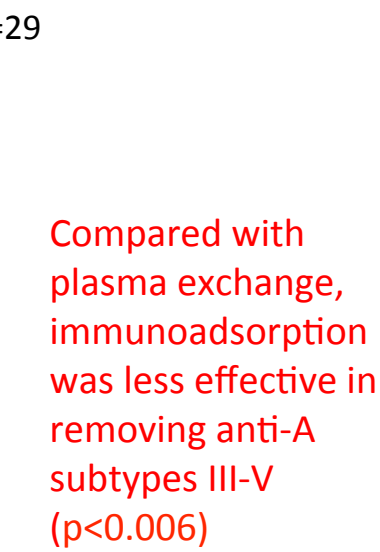

Anti-A subtype specific binding in 0 recipients of A-mismatched kidneys pre- and late-transplant (12 month)
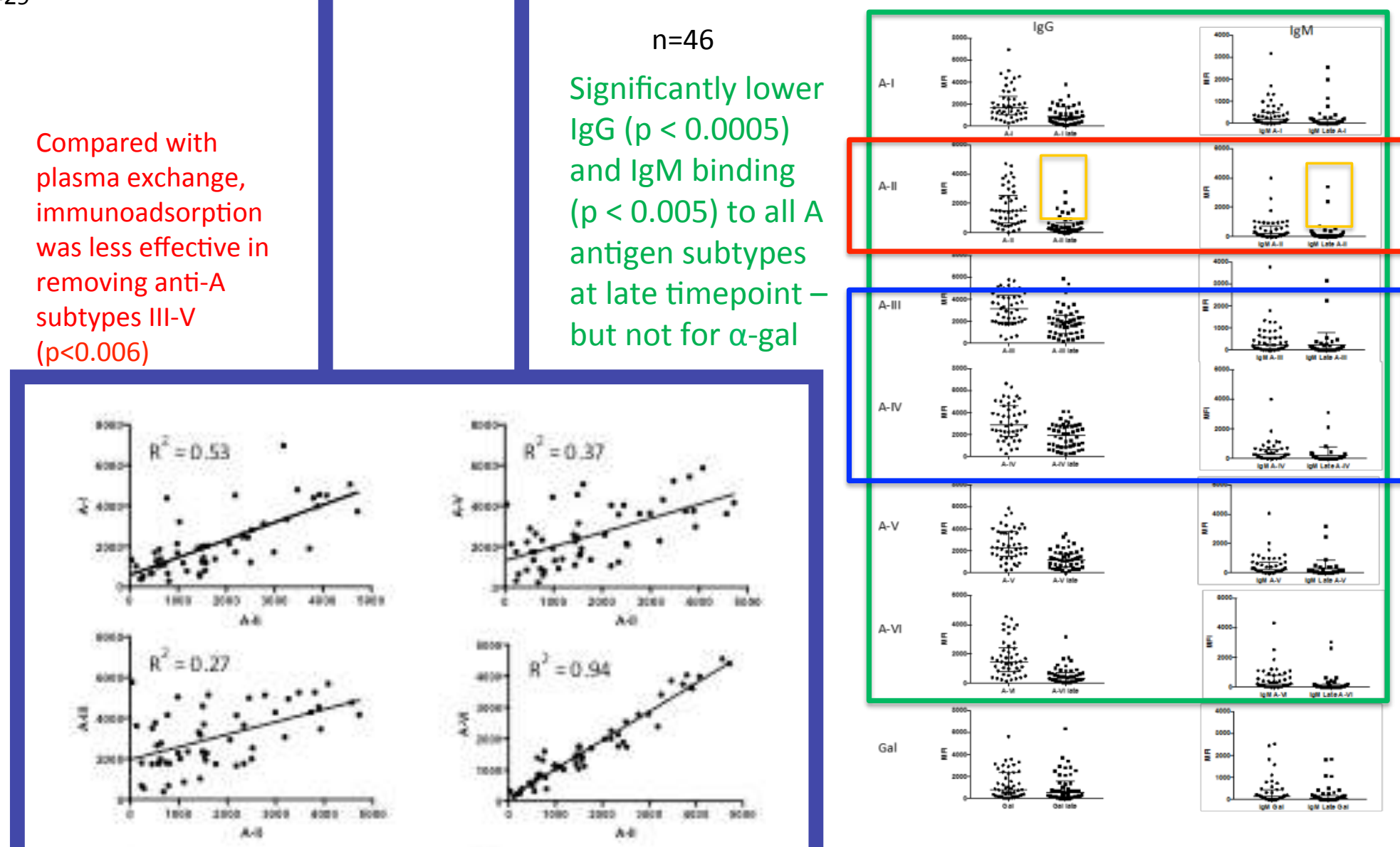

Most patients: $v$ low IgG/IgM anti-A-II antibodies at late timepoints ('tolerant' phenotype) $(p<0.005)$ to all $A$ antigen subtypes at late timepoint but not for $\alpha$-gal

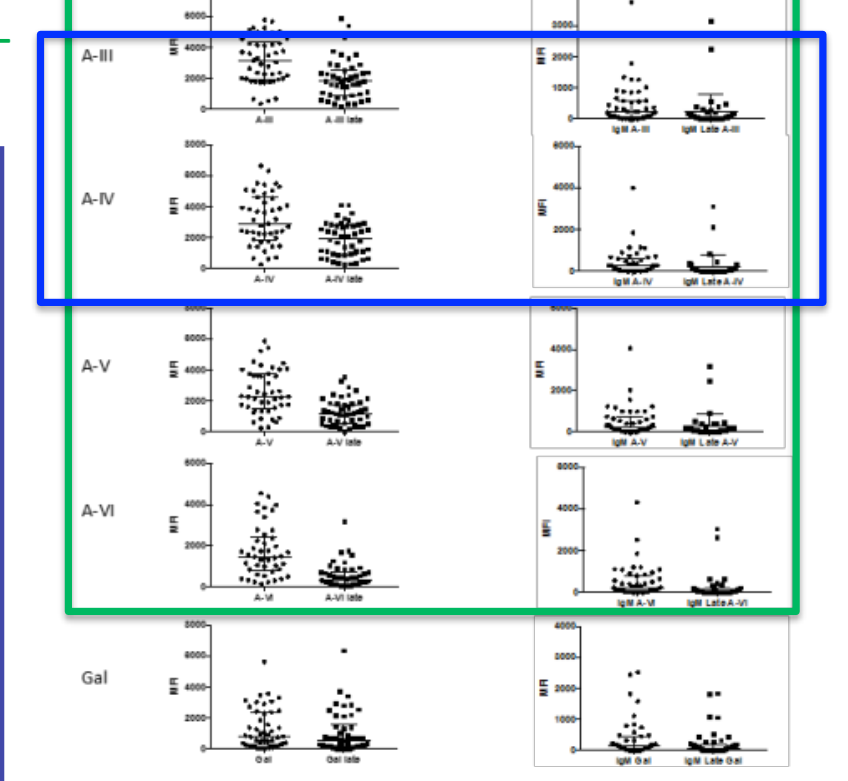

IgG Antibodies to AIII/IV subtypes clinical impact not yet clear

Reduction anti-A antibodies at late timepoints, but not anti-Gal. Anti-subtype antibodies were higher in late IgG, but low in IgM isotypes. IgG binding to subtype II was similar to VI.

\section{CONCLUSIONS}

These data demonstrate that IA preferentially removes IgG to subtype II compared to IgG to subtypes III/IV (subtypes for which there is evidence of that these tetrasaccharides contribute to epitope generation). They also indicate that accommodation, as well as modulation of antibody production, may be more important in adult ABOiKTx than in paediatric ABOiHTx, in which there is evidence that tolerance is the most important phenotype.

Potential mechanisms for accommodation in this setting are currently under investigation. 